\title{
THE IMPACT OF SHORT TERM TRAFFIC FORECASTING ON THE EFFECTIVENESS OF VEHICLES ROUTES PLANNING IN URBAN AREAS
}

\author{
Daniel Kubek, Paweł Więcek, Konrad Chwastek \\ Cracow University of Technology, Transport Section, Poland
}

\begin{abstract}
An impossibility to foresee in advance the accurate traffic parameters in face of dynamism phenomena in complex transportation system is a one of the major source of uncertainty. The paper presents an approach to robust optimization of logistics vehicle routes in urban areas on the basis of estimated short-term traffic time forecasts in a selected area of the urban road network. The forecast values of optimization parameters have been determined using the spectral analysis model, taking into account the forecast uncertainty degree. The robust counterparts approach of uncertain bi-criteria shortest path problem formulation is used to determining the robust routes for logistics vehicles in the urban network. The uncertainty set is created on the basis of forecast travel times in chosen sections, estimated by means of spectral analysis.

The advantages and the characteristics are exemplified in the actual Krakow road network. The obtained data have been compared with classic approach wherein it is assumed that the optimization parameters are certain and accurate. The results obtained in the simulation example indicate that use of forecasting techniques with robust optimization models has a positive impact on the quality of final solutions.
\end{abstract}

\section{INTRODUCTION}

The basic and natural feature of the transport system is dynamism of phenomena and processes, and the dynamism is a consequence of the transport system properties: high level of complexity (large-scale system), large number of users (diverse transport interactions and behaviours), occurrence of complex and random traffic phenomena and susceptibility to external factors (weather, reliability of technical and road infrastructure) (Adamski,2003;Kubek 2015). High dynamism and randomness of processes and phenomena in the city transport system does influence the quality of obtained data and information which describe this system. Such quality can be expressed, inter alia, by means of so-called uncertainty and indeterminacy of data. The uncertainty of traffic information can be understood as an impossibility to foresee in advance the detailed traffic parameters which would not have any errors. For example, using historical data to plan future, real processes will always have a certain forecast error. Such error forms an uncertainty set for the described process. On the other hand, the indeterminacy of information is rooted in limitations which result from the technical and economic possibilities of conducting the traffic measurements. For instance, a traffic study in an area of the city is performed in a selected few typical and 
representative points. The measurements are not performed for the remaining elements of this area, i.e. road section, intersection, intersection inlet or lane. Absence of such data causes indeterminacy of the obtained information. The missing data are often supplemented by mathematical models which have estimation errors, just like in case of forecasting methods. Hence, the second basic source of uncertainty of traffic information is forecasting models used to estimate future traffic parameters. The other source of uncertainty of traffic information is the accuracy of implementation of a solution in practical conditions (Ben-Tal and El Ghaoui and Nemirovski, 2009).

These premises indicate that the correct approach to describe the actual objects is methods which allow to define the uncertain parameters, i.e. the parameters which can assume values from a given range. There are a few such approaches in the literature now. These are, inter alia, stochastic optimization, optimization in which the variables are expressed by fuzzy sets, and the robust optimization. The first two are rather popular methods to cope with uncertain data, but their basic shortcoming in application to actual problems is the assumption that the randomness is implemented on the basis of known probability distribution. In case of urban traffic, sudden changes in travel time are natural, but it is not possible to indicate the probability distribution which could describe such changes. Examples would be random traffic accidents, traffic incidents, roadworks, breakdown of the control system. These are so-called non-recurrent incidents which are difficult or even impossible for foresee.

The third approach - robust optimization - is a method to account for data uncertainty, however here the variability is not described with a single, specific probability distribution (El Ghaoui, 2003; Bertsimas, and Brown and Caramanis, 2008). This solution is resistant to the fluctuations of model parameters when - for many parameter value scenarios - the obtained solution is at least acceptable and meets all the model conditions (model limitations) with high probability. The cost of such solution is the least of the worst possible variants.

On the basis of aforementioned issues, it seems justified to develop and analyze the models describing the transport processes where the model parameters can change their values. Further in the paper, presented is the issue of short-term traffic forecasts - a key issue for the turban traffic control and management. Then, we deal with the subject of determining the optimum routes for logistics vehicles in the urban network using the robust optimization on the basis of forecast travel times in chosen sections, estimated by means of spectral analysis. The obtained data (results) have been compared with classic approach wherein it is assumed that the optimization parameters are certain and accurate (e.g. deterministic data). The advantages and the characteristics are exemplified in the actual Krakow road network.

\section{SHORT-TERM TRAFFIC PREDICTION MODELS}

\subsection{Review of Methods}

The short-term traffic forecasting in urban areas has become in the last decades an important subject of research in the transport engineering and the urban logistics systems. The interest is stirred mainly by the need to develop intelligent traffic control methods, create advanced passenger information systems and applications which facilitate the deliveries of goods in 
the municipal road network. The other reasons include growing traffic in urban areas and the development of data acquisition and processing technologies (Barceló, 2010; Herrera,2010; Treiber and Kesting, 2012; Vlahogianni and Golias and Karlaftis, 2004).Short-term traffic forecasting, as an integral part of the Intelligent Transportation Systems, relates mostly to predicting future values of parameters such as traffic volume, density, speed or travel time in sections of the urban road network. Such prediction has the time horizon spanning from a few seconds to maximum a few hours. The values are estimated usually on the basis of current and historical traffic information. Transport and logistics companies nowadays need to have forecasts which are resistant to shorter or longer changes in traffic conditions. Such necessity results from growing competition in the market and the desire to provide increasingly reliable transport services. Making traffic forecasts on the urban road network level is still a challenge, mainly due to difficulties to install sensors in an adequate portion of the streets and to find optimum locations for these sensors. Such problem is discussed at length in (Gentili and Mirchandani, 2012; Hu and Peeta, and ChunHsiao, 2009).

The review of foreign literature on short-term traffic forecasting shows an abundance of available methods and tools. Only the most often used methods and tools will be mentioned in this paper. A lot of attention in these publications is given to models based on nonparametric regression models (Clark, 2003; Smith and Williams and Oswald , 2002), Autoregressive Integrated Moving Average - ARIMA (Kamarianakis and Prastacos, 2005; Williams, 2001). Various smoothing and filtering techniques are also frequently applied, for example Kalman filter models (Guo and Williams, 2010; Xie and Yuanchang and Zhang and Ye, 2007). Large randomness and indeterminacy in the urban traffic result in popularity of approaches using Bayesian networks based on the probability theory (Fei and Lu and Liu, 2011; Ghosh and Basu and O'Mahony, 2007). These methods belong to the group of statistical analysis of time series which yield good results when the modelled phenomenon does not show sudden changes in time. When such sudden changes in traffic are present, which indeed is the case, the research on traffic forecasting methods focus on using the Artificial Intelligence tools such as models of artificial neuron networks, fuzzy reasoning systems, methods based on frequency analysis, and also the hybrid models. Thus, all advantages of these methods are used, making the forecasts more accurate and at the same time resistant to sudden changes in traffic. Examples of such solutions are presented with more detail in (Chan and Dillon and Singh and Chang, 2012; Dimitriou and Tsekeris and Stathopoulos, 2008; Vlahogianni and Karlaftis, 2013; Yin, 2002; Zhang and Zhang and Haghani, 2014).

\subsection{Using Spectral Analysis Techniques in Short-Term Traffic Forecasts}

Basic components of each time series include trend, constant level of variable, a cyclic component and a random component. This also applies to time series which describe the traffic data. In reality, by analysing data from individual sections of road network, in addition to random disturbances, a certain cyclicity can be observed (rush hours, specific days of the week or month, weekends, etc.). Such being the situation, using frequency analyses to make forecasts seems justified. The basic goal of spectral analysis is to notice the cyclicity of processes. The analysis assumes a waveform structure of variable stochastic processes, 
allowing to analyse the series in the frequency domain. This is possible due to trigonometric functions, sine and cosine, which are often called harmonics. The number of harmonics for $n$ observations is $n / 2$ (Osińska, 2006). The period of the first harmonic is $n$, second $-n / 2$, third - n/3. etc. Thus, the course of a given process can be presented as:

$$
Y_{t}=f(t)+\sum_{i=1}^{n / 2}\left[a_{i} \sin \left(\frac{2 \pi}{n} i t\right)+b_{i} \cos \left(\frac{2 \pi}{n} i t\right)\right]
$$

where

$i_{-}$harmonic number

$a_{1}, b_{1}, a_{2}, b_{2}, \ldots$ are constant values

$f(t)$ - function describing the trend occurring in the series

The values $a_{1}, b_{1}, a_{2}, b_{2}$ are calculated using the least squares method, according to the following formulas:

$$
\begin{aligned}
& a_{i}=\frac{2}{n} \sum_{i=1}^{n}\left[y_{t} \sin \left(\frac{2 \pi}{n} i t\right)\right] \text { for } \quad i=1 \ldots \frac{n}{2}-1 \\
& b_{i}=\frac{2}{n} \sum_{i=1}^{n}\left[y_{t} \cos \left(\frac{2 \pi}{n} i t\right)\right] \text { for } \quad i=1 \ldots \frac{n}{2}-1
\end{aligned}
$$

For the last harmonic, it should be assumed that:

$$
\begin{gathered}
a_{n / 2}=0 \\
b_{n / 2}=\frac{1}{n} \sum_{i=1}^{n}\left[y_{t} \cos (\pi t)\right]
\end{gathered}
$$

Using the discrete Fourier transform of the analysed time series, we obtain the spectrum which is a function of frequency. The determined spectrum of the analysed process allows to decide which frequencies are capable of explaining the series variability to the greatest degree, i.e. the frequencies which have the greatest impact on the variance of the forecast variable (Zeliaś and Pawełek and Wanat, 2003). The part of the total variance of the variable explained by the ith harmonic is presented by the following formulas:

$$
\begin{gathered}
\omega_{i}=\frac{a_{i}^{2}+b_{i}^{2}}{2 \sigma^{2}} \text { for } i=1 \ldots \frac{n}{2}-1 \\
\omega_{i}=\frac{a_{i}^{2}+b_{i}^{2}}{\sigma^{2}} \text { for } i=\frac{n}{2}
\end{gathered}
$$

where: 
$\sigma^{2}$ - variance of the forecast variable with trend previously eliminated

\section{PROBLEM OF DETERMINING ROBUST PATHS}

The Shortest Path Problem (SPP) is a very well-known and popular problem in the operational research. Its goal is to find a path between any two points in the road network, using a criterion, usually the shortest distance. In the further analysis, the SPP has been formulated as an uncertain model in which the data can assume values from a given range. The variability degree of such data has been taken from the forecast of short-term speed value in individual sections of the road network and has been determined by means of the average error of the applied forecast models.

The uncertain models in their unmodified form, subjected to the optimization process, are often computationally intractable, meaning that a given mathematical model cannot be solved during polynomial computation time. References (Ben-Tal and El Ghaoui and Nemirovski, 2009; Bertsimas and Sim, 2003; Goh and Sim, 2010) present the robust optimization theory methods which allow to reformulate the uncertain problems to the robust counterparts of the original problem, which in turn are computationally tractable. Moreover, the reference (Ben-Tal and El Ghaoui and Nemirovski, 2009) proved that the proposed approach of replacing uncertain models with their robust counterparts gives better times of computer calculations than a deterministic model of a problem. One of the methods to modify the uncertain models is applying the duality theory and related theorems (the method was presented in (Bertsimas and Sim, 2004; Bertsimas and Sim, 2003)). The robust counterpart is created by using the stronger duality theorem which assumes that if an original problem or a dual problem has a solution, then the other also has a solution and the values of the objective function of both problems are equal (Bertsimas and Sim, 2003). The characteristics of this method as exemplified by the SPP is presented further in the paper.

The Robust Shortest Path Problem (RSPP) model was developed on the basis of (Bertsimas and Sim, 2004; Bertsimas and Sim, 2003) and previous research in (Kubek,2014a; Kubek, $2014 \mathrm{~b})$. The RSPP can be presented using the graph theory, where it is assumed that: there is a directed graph $\mathrm{G}=(\mathrm{V}, \mathrm{A})$, where $\mathrm{V}=\{1,2, \ldots, \mathrm{n}\}$ is the set of vertices, and $A=\{(i, j): i \wedge j \in V, i \neq j\}$ is the set of directed graph arcs. To each arc $(i, j)$ from the set $A$ assigned were weights: travel time $\mathrm{T}$ and the arc length $\mathrm{D}$. It is assumed that the travel time can take values from the set $\mathrm{T}=\left\{\mathrm{T}_{\mathrm{ij}} \in\left[\mathrm{T}_{\mathrm{ij}}{ }^{\text {med }}-\mathrm{T}_{\mathrm{ij}}{ }^{\text {dev }}, \mathrm{T}_{\mathrm{ij}}{ }^{\text {med }}+\mathrm{T}_{\mathrm{ij}}{ }^{\text {dev }}\right], \forall(\mathrm{i}, \mathrm{j}) \in \mathrm{A}\right\}$, where $\mathrm{T}_{\mathrm{ij}}{ }^{\text {med }}$ means the expected travel time, and $\mathrm{T}_{\mathrm{ij}}{ }^{\mathrm{dev}}$ means the possible deviation from the expected travel time. The problem is to find the optimum connection between two points in the network: $\{$ org,des $\} \subseteq \mathrm{V}$, in terms of the used criterion, taking into account some degree of data uncertainty. The criterion comprises two aspects: path length expressed as the travel time, and the distance travelled. The goal is to find the path in the network which has the shortest time and length. There are the following variables in the model:

- $x_{i j} \in\{0,1\}$ - means whether the arc $(\mathrm{i}, \mathrm{j})$ is in the path,

_ $y_{i j} \geq 0$ - additional variable which corresponds to the optimal solution of the original problem (uncertain model). 
_ $\alpha_{i j} \geq 0$ - additional dual variable, resulting from the linearization of the SPP uncertain model,

- $\theta \geq 0$ - additional dual variable, resulting from the linearization of the SPP uncertain model.

The criterion function of the RSPP model consists of two members: minimization of time and minimization of distance, and can take the following form:

$$
\begin{gathered}
\min \left[Q \cdot \frac{F_{1}-F_{1}^{\min }}{F_{1}^{\max }-F_{1}^{\min }}+(1-Q) \cdot \frac{F_{2}-F_{2}^{\min }}{F_{2}^{\max }-F_{2}^{\min }}\right] \\
F_{1} \geq \sum_{(i, j) \in A} x_{i j} \cdot T_{i j}+\theta \cdot \Gamma+\sum_{(i, j) \in A} \alpha_{i j} \\
F_{2} \geq \sum_{(i, j) \in A} x_{i j} \cdot D_{i j} \\
\sum_{\{j:(i, j) \in A\}} x_{i j}-\sum_{\{j:(i, j) \in A\}} x_{j i}=\left\{\begin{array}{cc}
1 \quad d l a \quad i=o r g \\
-1 \quad d l a \quad i=d e s \\
0 \quad \text { w.p.p }
\end{array}\right. \\
\theta+\alpha_{i j} \geq T_{i j}^{d e v} \cdot y_{i j}, \quad \forall(i, j) \in A \\
-y_{i j} \leq x_{i j} \leq y_{i j}, \quad \forall(i, j) \in A \\
x_{i j}=\{0,1\}, \quad \forall(i, j) \in A \\
\forall(i, j) \in A \quad \alpha_{i j} \geq 0, \quad \forall(i, j) \in A \theta \geq 0
\end{gathered}
$$

The criterion function (8) has been written in the normalized form. The normalization has been introduced due to the fact of adding two different criteria with varying units of measure. Parameters $\mathrm{F}_{1}{ }^{\min }, \mathrm{F}_{1}{ }^{\max }$ mean respectively the minimum/ maximum travel time in a given network, and parameters $\mathrm{F}_{2}{ }^{\min }, \mathrm{F}_{2}{ }^{\max }$ - respectively the minimum/ maximum length of path in a given network where the distance was the criterion. The additional parameter $\mathrm{Q}$ was introduced in order to be able to assign appropriate weights to a given member of the criterion. The limitation (9) corresponds to the criterion of time in which the travel time uncertainty was accounted for. In addition to dual variable, the inequality also includes a new parameter $\Gamma$ which in the literature on the subject matter is interpreted as level of conservatism of the obtained solution. In practice, it determines the number of coefficients of criterion function costs matrix for which there are deviations from the average value. The expression (10) is the second model criterion - minimization of the total path length (11). Limitations (12) and (13) relate to the dual variables which guarantee the correctness of the robust solution of the original problem. The last limitations result from determination of the type of variables.

This model accounts for data fluctuations expressed by travel time. The main goal of such formulation of the SPP is to find such path between any two points for which the travel time will be the least of the worst possible scenarios, with simultaneous minimization of the route 
distance. This is not, however, a classic min-max approach as the deviation value is controlled by the parameter $\Gamma$. Its value can be changed in real decision-making process, and the applied value should depend on the subjective views of the decision maker. For example, when the weather deteriorates, it can be assumed that the traffic flow will be reduced, so the level of uncertainty of the travel time will increase, hence $\Gamma$ should be greater.

\section{CASE STUDY}

\subsection{Assumptions and Data}

The models presented in previous arts of the paper have been used to check what advantages and disadvantages can be obtained by using robust models in determining the paths/ routes in a city. Currently, to determine routes of their vehicles some transport companies use computer software or portals where one can check the foreseen travel time. However, as it was already mentioned, the transport system is dynamic, and the forecasting models included in the software or portals have their forecasting error. The possibility to change the travel time is not accounted for, which can be bring notable benefits to transport companies, as this paper proves.

The analysis was performed for the real road network in Krakow, Poland (Figure 1). As the continuous traffic measurement is not implemented, the traffic data were generated in the traffic simulator AIMSUN 8.0. This software allows a micro-simulation of road traffic. The simulations were calibrated with actual traffic detection performed by induction loops installed in Krakow's road network which provided data on hourly number of vehicles during a day, for one full week, from 72 induction loops in total. In the analysis we used four days of the week, Monday, Tuesday, Wednesday, Thursday, and the time was limited to 15 hours, i.e. from 7.00 to 21.00. The remaining hours are night-time, hence taking them into account does not make much sense. A traffic micro-simulation was made for each hour on the basis of calibrated O-D (origin - destination) matrix. The level of fit of simulation results and actual data was established by a coefficient of determination. Its average value was $78.2 \%$ and it fluctuated in the $[70 \%, 86 \%]$ range. The data obtained from the simulator were accepted as actual data, and then used as input in our analysis.

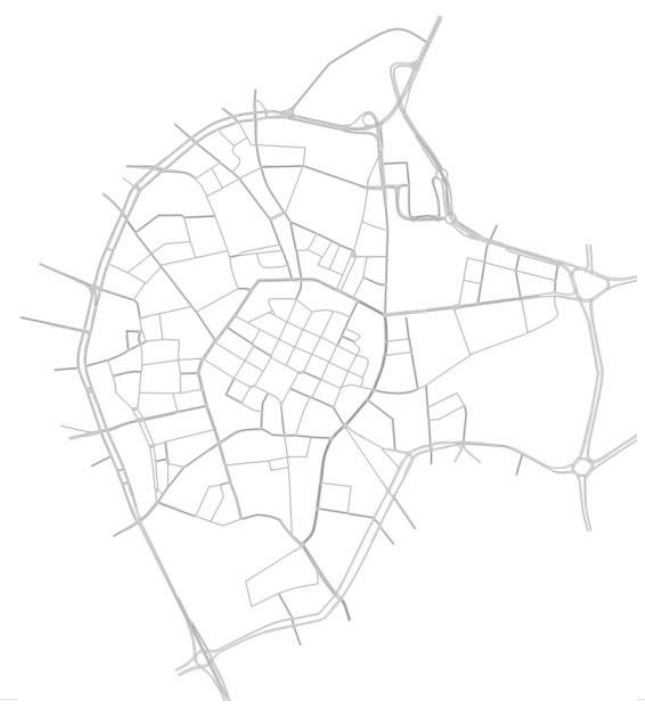




\section{Figure 1 Analysed Krakow's road network.}

The actual data were used to check the accuracy level of the forecasting model based on the spectral analysis of time series. The model was trained on the data from the first three days, and then a forecast was made for the next period, i.e. one hour for each section of the road network, that is for 685 sections. The forecast error was determined as a relative average error in relation to the actual value. In this case, the average error was $24.29 \%$, but for the half of the sections the error was below $12 \%$. In further investigation, the average forecast error for every sections was used to create a set of travel time uncertainties in the RSPP model. Typical values of forecast travel time for a given section in comparison with actual data are given in Figure 2.

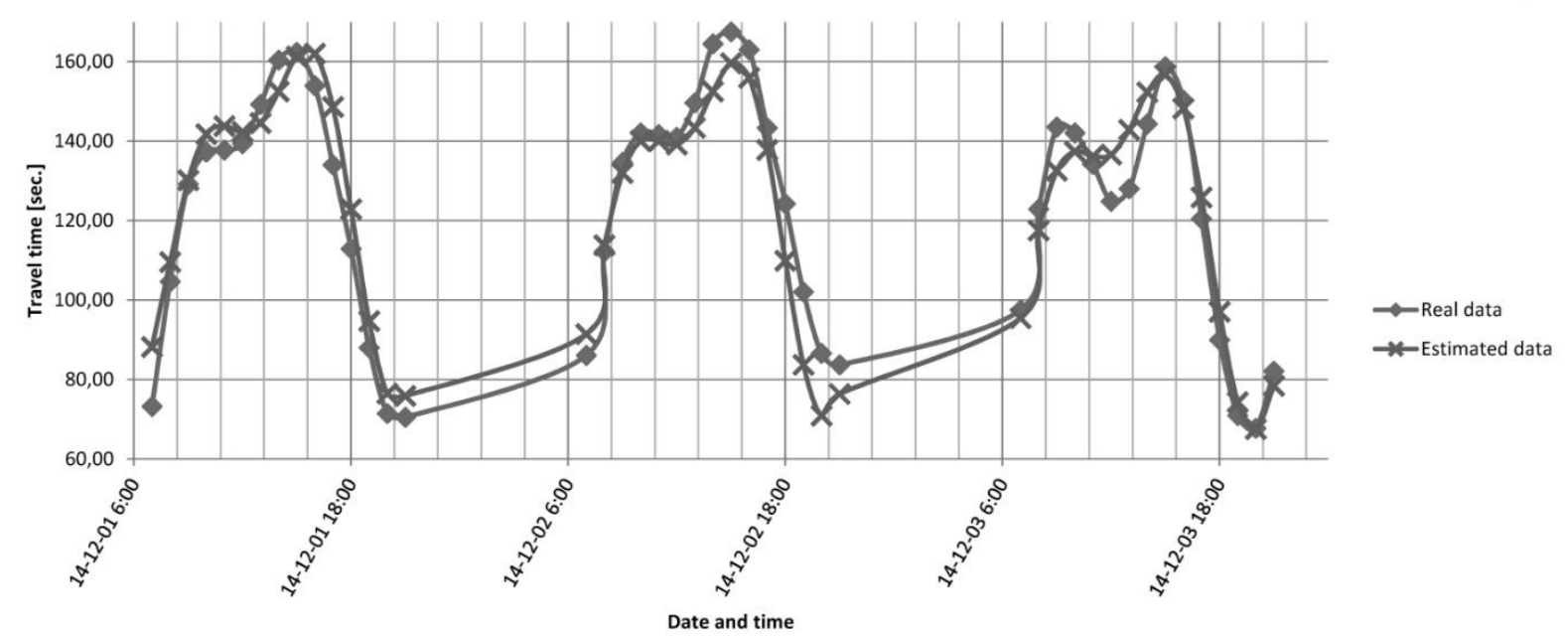

Figure 2 Typical travel time variability obtained from the AIMSUN simulator in comparison with the forecast data.

\subsection{Simulation Results}

In order to present the obtainable effects of using uncertain models and robust optimization of the shortest path problem, we calculated 90 paths for randomly selected pairs of points (network intersections) on the basis of deterministic data and data which include uncertainty. The deterministic paths are to show the situation which currently occurs in transport companies - determining the vehicle routes from the data which are accurate by definition. In the other simulation, we determined the paths for the same pairs of points according to the presented RSPP model where the average forecast error established the set of uncertainties. In both cases the weight of sub-criteria $Q$ was $60 / 40 \%$ for the time and the path length, respectively. This ratio was indicated by one of the leading transport companies which provides global transport and logistics services. The level of conservatism was set to $\Gamma=10$. The calculations are to show that despite using advanced forecasting methods, the differences in paths routing significantly affect the final costs. Please note that the presented simulations do not account for the dynamism of the transport system and non-recurrent situations, i.e. the road incidents. The changeability in travel time values is assumed to be an 
uncertainty set created only by forecasting model. The obtained results are presented in Table 1.

Table 1 Positive and negative aspects of optimization for the SPP

\begin{tabular}{|l|r|r|r|r|r|r|r|r|}
\hline \multirow{2}{*}{ Reduction } & \multicolumn{4}{|c|}{ Travel time } & \multicolumn{4}{c|}{ Distance } \\
\cline { 2 - 9 } & Amount & Min. & Average & Max. & Amount & Min. & Average & Max. \\
\hline Positive & 19 & $1,84 \%$ & $11,25 \%$ & $24,55 \%$ & 6 & $3,27 \%$ & $4,74 \%$ & $6,50 \%$ \\
\hline Negative & 0 & $0,00 \%$ & $0,00 \%$ & $0,00 \%$ & 13 & $-22,16 \%$ & $-7,90 \%$ & $0,00 \%$ \\
\hline Neutral & 71 & $0,00 \%$ & $0,00 \%$ & $0,00 \%$ & 71 & $0,00 \%$ & $0,00 \%$ & $0,00 \%$ \\
\hline
\end{tabular}

Out of 90 cases, over $21 \%$ of robust paths have shown a sufficient resistance to changes of travel time, which resulted in a positive reduction. The reduction here is understood as a difference between the cost of the deterministic solution and the cost of the robust solution, in relation to the former. The robust solution has caused losses in distance in about $2 / 3$ cases but the extension was lower than possible travel time profits. Most of solutions have neither increased costs nor generated costs in the form of extended time or distance. To sum up this analysis, a rather intuitive conclusion can be drawn: bypassing traffic jams or bottlenecks in road network results in higher costs relating to the path length. However, in the light of shifting priorities of transport companies toward better customer service and service quality, the possibility of increasing the effectiveness of the be parameters can be interesting.

\section{SUMMARY}

The problem dealt with in the paper indicates that despite many advanced methods of shortterm urban traffic forecasting, there is a clear need for using robust models to determine the routes of vehicles which provide transport services inside cities. High variability and indeterminacy is a natural feature of traffic, particularly in urbanized areas. Hence, in order to obtain more effective and reliable data it makes sense to account for uncertainty of forecast data while planning the routes for urban logistics vehicles. The results obtained in the calculation example indicate that use of forecasting techniques with robust optimization models has a positive impact on the quality of final solutions. The approach presented in the paper can be used with success in practical operation of transport and logistics companies.

\section{REFERENCES}

1. ADAMSKI A. (2003) Inteligentne systemy transportowe: sterowanie, nadzór i zarządzanie. Wydawnictwo AGH, Kraków.

2. BARCELÓ, JAUME et al (2010) Travel time forecasting and dynamic origin-destination estimation for freeways based on bluetooth traffic monitoring. Transp.Res. Rec.: J. Transp. Res. Board. 2010. 2175 (1). P. 19-27.

3. BEN-TAL A. \& EL GHAOUI L. \& NEMIROVSKI A. (2009) Robust optimization. New Princeton University Press, Jersey.

4. BERTSIMAS, D. \& BROWN D.B. \& CARAMANIS C. (2008) Theory and Applications of Robust Optimization. SIAM Review, Tom 53(3).

5. BERTSIMAS, D. \& SIM M. (2004) Price of Robustness. Operations Research, Tom 54(1) 
6. BERTSIMAS, D. \& SIM M. (2003) Robust discrete optimization and network flows. Mathematical Programming, Tom 98,

7. CHAN, K.Y. \& DILLON, T.S. \& SINGH, J. \& CHANG, E. (2012) Neural-network-based models for short-term traffic flow forecasting using a hybrid exponential smoothing using a hybrid exponential smoothing and levenberg-marquardt algorithm. IEEE Transactions on Intelligent Transportation Systems. 2012a. 13 (2), P. 644-654.

8. CLARK, STEPHEN (2003) Traffic prediction using multivariate nonparametric regression. $J$. Transp. Eng. 129 (2), P. 161-168.

9. DIMITRIOU, L. \& TSEKERIS T. \& STATHOPOULOS, A. (2008) Adaptive hybrid fuzzy rule-based system approach for modeling and predicting urban traffic flow. Transportation Research Part C: Emerging Technologies. 16 (5), P. 554-573.

10. EL GHAOUI L. (2003) Uniwersytet w Berkeley. Robust Optimization and Applications. [Online] [Zacytowano: 19 maj 2015]

11. FEI, X. \& LU, C.-C. \& LIU K.A. (2011) Bayesian dynamic linear model approach for realtime short-term freeway travel time prediction. Transportation ResearchPart C: Emerging Technologies. Vol. 19 (6) P. 1306-1318.

12. GENTILI M. \& MIRCHANDANI P.B. (2012) Locating sensors on traffic networks: models, challenges and research opportunities. Transportation Research Part C: Emerging Technologies. Vol.24. P. 227-255.

13. GHOSH, B. \& BASU B. \& O’MAHONY M. (2007) Bayesian time-series model for short-term traffic flow forecasting. Journal of Transportation Engineering. 133 (3). P. 180-189.

14. GOH J. \& SIM M. (2010) Distributionally Robust Optimization and Its Tractable Approximations. Operations Research. Tom 58(4)

15. GUO J. \& WILLIAMS B.M. (2010) Real-time short-term traffic speed level forecasting and uncertainty quantification using layered Kalman filters. Transportation Research Record. Vol. 2175. P. 28-37

16. HERRERA JUAN C. et al. (2010) Evaluation of traffic data obtained via GPS-enabled mobile phones: The Mobile Century field experiment. Transp. Res. Part C: Emerg. Technol. Vol. 18 (4), P. 568-583.

17. HU, SH.-R. \& PEETA, S. \& CHUN-HSIAO CHU. (2009) Identification of vehicle sensor locations for link-based network traffic applications. Transportation Research Part B: Methodological. Vol. 43 (8-9), P. 873-894.

18. KAMARIANAKIS Y. \& PRASTACOS, P. (2005) Space-time modeling of traffic flow. Comput. Geosci. Vol. 31 (2), P. 119-133.

19. KUBEK D. (2014b)Wyznaczanie odpornych ścieżek w warunkach miejskich, Czasopismo Logistyka [CD]. Nr 4. P. 3965-3971.

20. KUBEK D. (2015) Zastosowanie optymalizacji odpornej w problematyce wyznaczania tras pojazdom - charakterystyka oraz kierunki badań, Czasopismo Logistyka. nr 3, [w druku]

21. KUBEK D. (2014a) Wyznaczanie tras typu „robust” dla pojazdów logistyki miejskiej. Czasopismo Logistyka [CD]. Nr 3, P. 3461-3470

22. OSIŃSKA M.(2006) Ekonometria Finansowa. PWE. Warszawa.

23. SMITH BRIAN L. \& WILLIAMS, BILLY M. \& OSWALD R. KEITH. (2002) Comparison of parametric and nonparametric models for traffic flow forecasting. Transp. Res. Part C: Emerg. Technol. Vol. 10 (4). P. 303-321.

24. TREIBER, M. \& KESTING, A. (2012) Validation of traffic flow models with respect to the spatiotemporal evolution of congested traffic patterns. Transportation Research Part C: Emerging Technologies. Vol. 21 (1), P. 31-41

25. VLAHOGIANNI E.I. \& GOLIAS, J.C. \& KARLAFTIS M.G. (2004) Short-term traffic forecasting: overview of objectives and methods. Transportation Reviews. Vol. 24 (5). P.533557.

26. VLAHOGIANNI, E.I. \& KARLAFTIS M.G. (2013) Testing and comparing neural network and statistical approaches for predicting transportation time series. Transportation Research Record Journal of the Transportation Research Board.Vol 2399 
27. WILLIAMS BILLY M. (2001) Multivariate vehicular traffic flow prediction: evaluation of ARIMAX modeling. Transportation Research Record Journal of the Transportation Research Board. 1776 (1), P. 194-200.

28. XIE Y.\& YUANCHANG \& ZHANG Y. \& YE Z. (2007) Short-term traffic volume forecasting using Kalman filter with discrete wavelet decomposition. Comput.- Aid. Civ. Infrastruct. Eng. Vol. 22 (5), P. 326-334.

29. YIN, HONGBIN et al. (2002) Urban traffic flow prediction using a fuzzy-neural approach. Transportation. Research. Part C.Vol. 10 (2). P. 85-98.

30. ZELIAŚ A. \& PAWEŁEK B. \& WANAT S. (2003) Prognozowanie Ekonomiczne. PWN, Warszawa.

31. ZHANG Y. \& ZHANG Y. \& HAGHANI A. (2014) A hybrid short-term traffic flow forecasting method based on spectral analysis and statistical volatility model. Transportation Research. Part C. Vol. 43. P. 554-573. 